\title{
Irreversibility Analysis of R407C, R404A, and R134A as an Alternatives of R22 in Vapor Compression Chiller under Cycling Conditions
}

\author{
Ayad Khudhair Al-Nadawi* \\ Middle Technical University (MTU), Institute of Technology-Baghdad, Iraq. \\ E-mails: ayadalnadawi@gmail.com \\ ayadkhudhair@mtu.edu.iq
}

Received 20 September 2020, Accepted 24 February 2021

\begin{abstract}
This paper presents irreversibility analysis using experimental data from vapor compression chiller system using R22, R407C, R404A and R-134A as working fluids. The system operated under cycling condition, which allowing the water to circulate in the evaporator and recording data every ten minutes. Further, the experimental study was conducted at different water and ambient temperature to identify the parameter that cause the energy deterioration. The findings show that the total irreversibility increases at high water mass flow rate. Additionally, high ambient temperature increase the irreversibility of the system. R134A and R407C are a good replacement for R22 in terms of irreversibility analysis during cycling condition.
\end{abstract}

Keywords: R407C; R404A; R134A; Vapor Compression Chiller; Irreversibility Analysis.

\section{Introduction}

The refrigeration industry has experienced immensely changes since chlorofluorocarbons (CFCs) and hydrochlorofluorocarbons (HCFCs) were identified as a source of ozone layer depletion. CFCs and HCFCs refrigerants have phased out in an increasing pace since the signature of Montreal protocol in September 1987. According to that protocol, HCFCs were expected to phase out by 2030 and 2040 for developed and developing countries, respectively [1].

Water Chillers have been used widely in residential and industrial applications to provide cold water. The majority of the systems operate with refrigerant $\mathrm{R} 22$. The alternatives for R22 include hydrofluorocarbon (HFCs). Most of them are mixtures of two or three refrigerants.

For the past decade, R407C and R404A have been identified as a promising candidates for R22 and R134A, due to their performance potential, low ODP, preferable thermodynamic properties, and for easy retrofit of the old equipment.

Most of the previous research focused on Energy (first law) analysis which is still the most commonly used method in the analysis of refrigeration systems. The first law is concerned only with the quantity of energy. It does not determine where, how and the amount of performance degradation that could happen in the system as a whole or part of it.

Today's energy interests make it very important to analyze refrigeration equipment using the second law of thermodynamics. This approaches or irreversibility analysis deals with the quality of energy which allow engineers to reexamine the refrigeration cycle and new working fluids with respect to cycle losses and the cycle component inefficiencies[2].

Irreversibility analysis is based on both the first and second laws of thermodynamics to improve of the systems.
Irreversibilities in refrigeration cycle causes the system performance to degrade and must be minimized in order to increase a cycle's efficiency. The major source of irreversibility is heat transfer between the system and the surrounding which happens at a finite temperature difference. The performance losses need to be evaluated in all part of the cycle. In other words, irreversibility analyses can be used to determine both the magnitude and location of energy that is destroyed. For all of the above mentioned reasons, irreversibility analysis can provide a deeper insight into the process in terms of its performance losses.

Most of the previous studies focused on alternative refrigerants to $\mathrm{R} 22$ at steady state condition.

(Al-Nadawi, 2019) [2], presented irreversibility analysis for window type air conditioner unit. R407C, R407A, and R22 were studied. Irreversibilities was compared with different volumetric flow rates of the air passing through evaporator and different ambient temperatures. The results show that R22 has the highest irreversibility while R407A has the lowest one.

(Zhang et al., 2018)[3], proposed the exergy and energy analysis for $4 \mathrm{~kW}$ double evaporating traditional watercooled chiller using zeotropic mixture refrigerants of R32 and R236fa. The results prove that the chiller achieves the lowest exergy loss and highest exergy efficiency when the mass concentration ratio is $40 \%$ for R32 and $60 \%$ for R236fa.

(Mota-Babiloni et al., 2018)[4], concluded that the R513A global exergy efficiency is $0.4 \%$ higher than that of R134a. Also, there is a slight reduction in efficiency in the condenser using R513A.

(Tang et al., 2016)[5], Performed energy and exergy analysis of a refrigeration system with vapour injection. Results indicate that injection system can reduce exergy loss system, especially the compression exergy destruction. 
(Ben Jemaa et al., 2016)[6], introduced energy and exergy analysis to an air-cooled vapor compression chilled water using R1234ze and R134a. The results show that the irreversibility using R1234ze is lower than that of R134a.

(Dudar et al., 2013)[7], theoretically studied the possible exergy efficiency increase of two-phase ejector refrigeration compression cycle with various working fluids and various operating conditions. R134a, R507, R290, R600a, and R717 were used as working fluids. The authors demonstrated that, even for low-pressure refrigerants, there is improvement of the efficiency of the compression refrigeration cycle, which attributed to the complete reduction of the exergy losses occurring in the throttling valve and low losses produced in the compressor.

(Fukuda et al., 2012)[8], are experimentally tested the binary mixture R32/R1234yf and the ternary mixture R744/R32/R1234yf in water heat source vapor compression and compared to that of R410A. The results show that irreversible loss in heat exchanger increases because of lower heat transfer performance of heat exchanger.

(Ahamed et al., 2011)[9], has performed exergy analysis in compression refrigeration system using R600, R600a, and R290. It is found that R600a have $50 \%$ higher exergy efficiency than R134a.

(Stanciu et al., 2011)[10], analyses, in terms of exergy destruction, five refrigerants (R22, R134a, R717, R507a, and $\mathrm{R} 404 \mathrm{a})$ in one stage vapor compression refrigeration system. The results prove that $\mathrm{R} 717$ presents the highest value of irreversibilities. Further, the highest exergy destruction rate is in the compressor for R134A, R717 and almost for R22.

(Shilliday, Tassou and Shilliday, 2009)[11], presented energy and exergy analysis of R744 and R290 and compared with R404A in a single-stage vapour compression cycle and R744 in a two-stage vapour compression cycle with an internal heat exchanger. The results show that, for a singlestage cycle, both R404 and R290 exhibit higher COPs when compared with R744. R744 showed both the highest total cycle exergy ratio and the greatest increase in exergy ratio with increasing condensing temperature and evaporating temperature.

(Liang and Kuehn, 1991)[12], analyzed the irreversibility sources of a steady-flow, vapor-compression, water-to-water heat pump. Additionally, the irreversibilities due to heat transfer across a finite temperature difference and mass flow across a finite pressure drop in each component are calculated according to the second law of thermodynamics. The results prove that the increase in the heat-transfer coefficient of the heat exchangers will reduce the irreversible loss substantially.

Most of the previous studies considered only the irreversibility analysis with steady state condition. However, the present investigation is different from the previous studies because it presents a detailed experimental comparative analysis of irreversibility for R22, R407C, R404A and R134A in vapor compression chiller under cycling conditions. Further, the temperature of water passing through the evaporator and ambient temperature were taken into consideration.

It is noticed from the open literature that different criteria are used to find the best pure or blend refrigerants, for instance, low ODP and GWP values, high COP, refrigerating power, low compressor consumption, exergetic efficiency, safety, etc.

To enhance our understanding to the irreversibility behavior of the system under cycling conditions, three refrigerants were introduced to be tested as alternative refrigerants to R-22 in vapor compression chiller system. These refrigerants are zeotropic blend R-407C comprising of (R-32/125/134a) with mass percentages of (23/25/52) respectively, near-azeotropic blend R-404A consisting of (R125 / R143a / R134a) with (44/52/4) mass fraction percentage and finally R134a. The main difference between the pure, zeotropic, and near-azeotropic refrigerants is the difference in boiling point of their component due to the difference of vapour and liquid mass fraction at equilibrium [13]. Table 1 compares some characteristics of selected and common refrigerants.

Table 1. Comparing properties of different refrigerants.

\begin{tabular}{|c|c|c|c|c|c|c|c|c|}
\hline \multirow{2}{*}{ 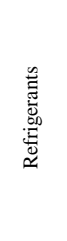 } & \multirow{2}{*}{ 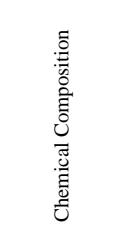 } & \multirow{2}{*}{ 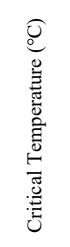 } & \multirow{2}{*}{ 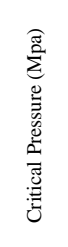 } & \multirow{2}{*}{ 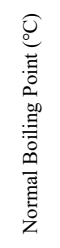 } & \multicolumn{2}{|c|}{ 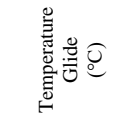 } & \multirow{2}{*}{ ชิ } & \multirow{2}{*}{ 恿 } \\
\hline & & & & & 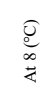 & 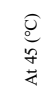 & & \\
\hline R22 & $\mathrm{CHClF}_{2}$ & 96.1 & 4.99 & -29.7 & 0 & 0 & 0.055 & 1810 \\
\hline $\mathrm{R} 407 \mathrm{C}$ & $\begin{array}{l}\mathrm{CH}_{2} \mathrm{~F}_{2}, \\
\mathrm{CF}_{3} \mathrm{CHF}_{2}, \\
\mathrm{CH}_{2} \mathrm{FCF}_{3}\end{array}$ & 86.1 & 4.65 & -43 & 7.9 & 4.7 & 0 & 1800 \\
\hline R407A & $\begin{array}{c}\mathrm{CH}_{2} \mathrm{~F}_{2}, \mathrm{C}_{2} \mathrm{HF}_{5}, \\
\mathrm{CH}_{2} \mathrm{FCF}_{3}\end{array}$ & 83 & 4.54 & -45.5 & 5.5 & 4 & 0 & 2100 \\
\hline $\mathrm{R} 404 \mathrm{~A}$ & $\begin{array}{l}\mathrm{CHF}_{2} \mathrm{CF}_{3}, \mathrm{CF}_{3} \\
\mathrm{CH}_{2} \mathrm{~F}, \mathrm{CH}_{3} \mathrm{CF}_{3}\end{array}$ & 72.05 & 3.729 & -46.2 & 0.47 & 0.31 & 0 & 3922 \\
\hline R410A & $\underset{\mathrm{CHF}_{2} \mathrm{CF}_{3}}{\mathrm{CH}_{2} \mathrm{~F}_{2}}$ & 71.36 & 4.9 & -51.4 & 0.11 & 0.11 & 0 & 2088 \\
\hline R134a & $\mathrm{CH}_{2} \mathrm{FCF}_{3}$ & 101.06 & 4.059 & -26.1 & 0 & 0 & 0 & 1430 \\
\hline
\end{tabular}

In the present study, irreversibility analysis was carried out in vapor compression chiller under cycling condition. The experimental study was done with various ambient temperatures (ranging from $25^{\circ} \mathrm{C}$ to $49^{\circ} \mathrm{C}$ ) and water temperature passing through the evaporator (ranging from $22^{\circ} \mathrm{C}$ to $30^{\circ} \mathrm{C}$ ) to study the effects of the parameters that cause the energy deterioration in the system. Also, this research examines the emerging role of cycling conditions in the irreversibility behavior. Four refrigerants, namely R22, R407C, R404A and R134A, were charged into the system and then the measurements were collected.

\section{Experimental Setup and Test Methodology}

In this section, experimental setup, uncertainties of the measurement system, and test methodology will be introduced.

\subsection{Experimental Plant}

The experimental work of Mahmood [14] will be recalled. A schematic diagram of the refrigerant side and water side experimental apparatus are shown in Figure 1. and Figure 2., respectively.

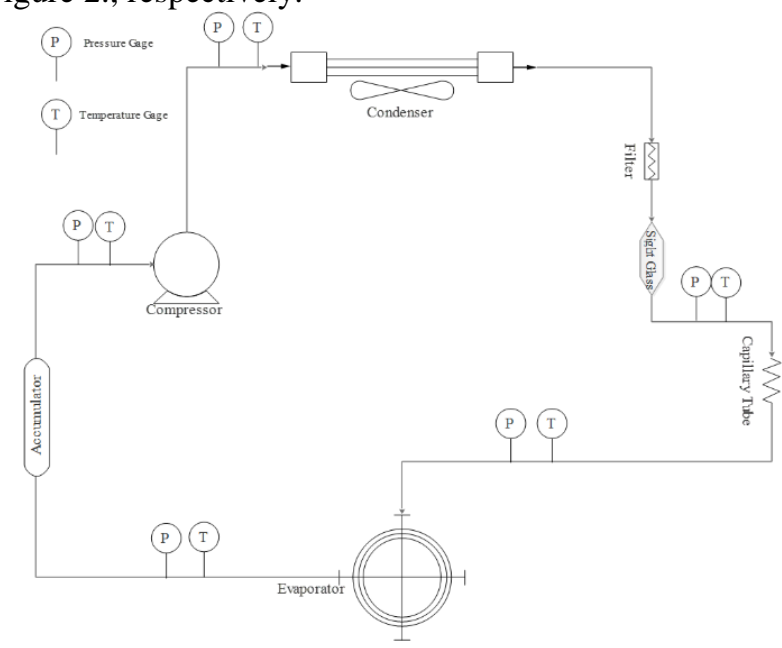

Figure 1. Schematic diagram of the refrigerant side. 
The refrigerant side of the test rig consists of:

1. Reciprocating hermetic compressor with polyol ester oil,

2. Finned tube condenser, Table (2) illustrate the characteristic and physical dimensions of the condenser,

3. Shell and coil evaporator, Table (3) illustrate the characteristic and physical dimensions of the evaporator,

4. One capillary tube $(900 \mathrm{~mm}$ length, and $1 \mathrm{~mm}$ inner diameter).

Table 2. The characteristic physical dimensions of the condenser, [14].

\begin{tabular}{cc}
\hline Dimension Specification & Condenser \\
\hline Tube Length $(\mathrm{mm})$ & 254 \\
Dimension $(\mathrm{L} \times \mathrm{W} \times \mathrm{H})(\mathrm{mm})$ & $254 \times 65 \times 279.4$ \\
No. of Circuits & 1 \\
No. of Rows & 3 \\
No. of Tubes & 30 \\
Tube outside diameter $(\mathrm{mm})$ & 9.52 \\
Tube inside diameter $(\mathrm{mm})$ & 7.93 \\
Tube material & Copper \\
Tube Metal Thermal conductivity & 386 \\
(W/m.K) & smooth \\
Inner Tube Surface & 25.4 \\
Transverse tube pitch (mm) & 22.225 \\
Longitudinal tube pitch $(\mathrm{mm})$ & 0.15 \\
Fin thickness (mm) & 2 \\
Fin pitch (mm) & Slit \\
Fin type & Aluminium \\
Fin metal & 202 \\
\hline Fin Thermal Conductivity & \\
(W/m.K) &
\end{tabular}

The condenser is made from copper tubes and slit fins, Table (2). The condenser has one circuit in three rows. Each row has ten copper tubes. The instruments of measurements details can be found in [14].

Table 3. The characteristic physical dimensions of the evaporator, [14].

\begin{tabular}{cc}
\hline Dimension Specification & Evaporator \\
\hline Shell material & Stainless steel \\
Thermal conductivity of shell & 15 \\
metal $(\mathrm{W} / \mathrm{m} . \mathrm{K})$ & \\
Tube material & Copper \\
Tube Metal Thermal conductivity & 386 \\
(W/m.K) & 20 \\
Shell volume (Litre) & $300 \times 300$ \\
Shell dimension (D× H) $(\mathrm{mm})$ & $250 \times 15$ \\
Coil tube dimension (D× L) $(\mathrm{mm})$ & 15 \\
Number of coil turns & 9.52 \\
Tube outside diameter $(\mathrm{mm})$ & 7.93 \\
Tube inside diameter $(\mathrm{mm})$ & smooth \\
Inner Tube Surface &
\end{tabular}

The water side of the test rig consists of:

1. Centrifugal type water pump with power, flow rate, head, and speed of 0.5HP, (5-30 $\left.\frac{\text { lit }}{\mathrm{min}}\right),(5.5-28 \mathrm{~m})$, and $\left(2900 \frac{\mathrm{rev}}{\mathrm{min}}\right)$, respectively, Figure 2 .

2. 85 liter water vessel with 2000 Watt electric heater.

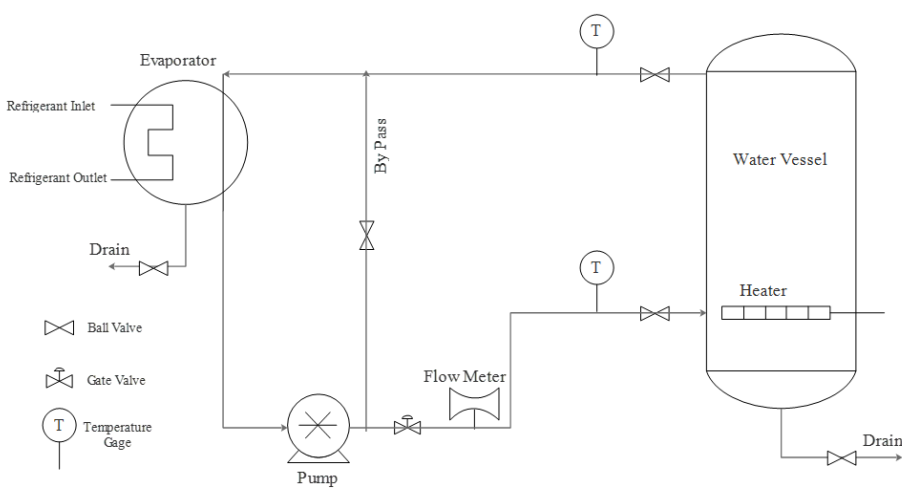

Figure 2. Schematic diagram of the water side.

The centrifugal type water pump, evaporator shell, and pipes are well insulated by Armflex (25 mm thickness and $\left.\mathrm{k}=0.036 \frac{\mathrm{W}}{\mathrm{m} \cdot \mathrm{K}}\right)$

\subsection{Measurement System and Uncertainties}

The test apparatus is fully instrumented in refrigerant side and water side to be able to measure parameters that affect the irreversibility. The data collected are:

1. Refrigerant temperature and pressure at inlet and outlet of the evaporator.

2. Refrigerant temperature and pressure at inlet and outlet of the condenser.

3. Refrigerant temperature and pressure at the suction line of the compressor.

4. Dry and wet bulb temperatures at inlet and outlet of the condenser.

5. Water temperature at inlet and outlet of the water vessel.

6. Water mass flow rate was fixed at $400 \frac{\mathrm{lit}}{\mathrm{hr}}$.

7. The current and voltage are measured for the unit.

Furthermore, detailed experimental uncertainties was estimated from experimental data as described in (AlNadawi, 2019)[2]. The following equations were introduced to calculate the uncertainties for the experimental values:

$\mathrm{Q}=\mathrm{f}\left(\mathrm{Z}_{1} \ldots \ldots \mathrm{Z}_{\mathrm{n}}\right)$

$\left(\frac{u_{q}}{q_{e}}\right)^{2}=\sum_{i=1}^{N}\left(\frac{u_{z}}{z}\right)^{2}$

$\mathrm{U}=\mathrm{k} \mathrm{u} \mathrm{q}$

Where $u_{\mathrm{q}}$ is the standard uncertainty, $q$ is the best estimate quantity, and $\mathrm{u}_{\mathrm{z}}$ is the standard uncertainty of input quantity.

For $98 \%$ degree of confidence level ascribed to the true value of $\mathrm{Q}$ being inside the interval, $\mathrm{K}$ will be taken as 2.327 , [15]. From calibration it was found that the maximum error in temperature reading was $\pm 4^{\circ} \mathrm{C}$ and maximum error in pressure gauges was $\pm 0.034 \mathrm{Mpa}$. The maximum typical uncertainties were estimated to be $1.83233 \%$ for COP and $1.83264 \%$ for irreversibility.

\subsection{Test Methodology}

This paper uses archival data from [14] to examine the effect of cycling mode (quasi steady state) on the irreversibility behavior of alternative refrigerants. To begin the experimental process, the water pump set on to circulate the water around the water system, Figure 2. The amount of water was controlled by using valves and by pass valve. After a certain time, the temperature of water in the water loop will equalize. Then, the water chiller was turned on for at least thirteen minutes in order to achieve stability. This experiment was conducted under cycling condition in which, 
after approaching certain evaporator water temperature, the heater was turned off and valves were closed. Finally, data were gathered from the test rig every ten minutes, and therefore, about 192 measurements were acquired for each operative point.

\section{Energy and Irreversibility Analysis Equations}

The data reported previously are used to calculate the enthalpy and entropy in each of the points where pressure and temperature were obtained. The pressure-enthalpy (P-h) and the temperature entropy (T-s) diagrams of refrigeration system are shown in Figure 3.

A number of assumptions are considered:

1. The compression process is adiabatic.

2. The water temperature at the evaporator coil was assumed to be constant and homogenous at all tubes.

3. The air temperature at the condenser inlet and outlet was assumed to be constant and homogenous at all tubes.

4. The mass flow rate of refrigerant is constant at all parts of the test rig.

5. The enthalpy change in the capillary tube is negligible.
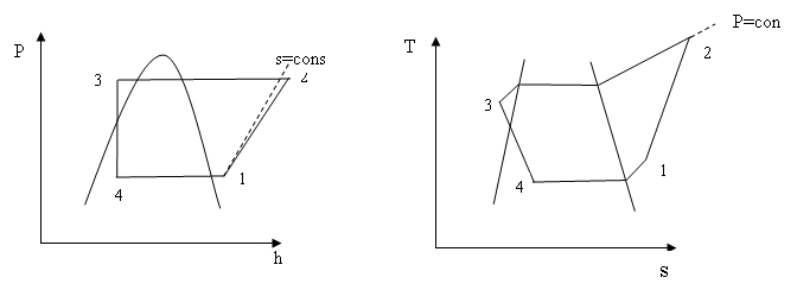

Figure 3. $P$-h diagram and $T$-s diagram for the refrigeration cycle, [2].

The refrigerant circulated mass flow rate can be calculated by.

$\dot{m}_{r}=\frac{m_{w} c_{p_{w}}\left(T_{w i}-T_{w o}\right)}{h_{1}-h_{4}}$

The energy and irreversibility equations for each component are summarized in Table 4.

Table 4. Energy and irreversibility equations for the water chiller cycle.

\begin{tabular}{ccc}
\hline & $\begin{array}{c}\text { Equations for energy } \\
\text { performance }\end{array}$ & $\begin{array}{c}\text { Equations for irreversibility } \\
\text { performance }\end{array}$ \\
\hline Compression & $\begin{array}{c}\text { The power factor }( \\
\text { cos } \emptyset \text { ) was assumed to } \\
\text { be unity. }\end{array}$ & $I_{\text {comp }}=\dot{m}_{r}\left(s_{2}-s_{1}\right)$ \\
& & $I_{e}$ \\
Evaporation & $Q_{e}=m_{w} C_{p_{w}}\left(T_{w i}\right.$ & $=\dot{m}_{r}\left[\left(s_{1}-s_{4}\right)\right.$ \\
& $\left.-T_{w o}\right)$ & $\left.-\frac{\left(h_{1}-h_{4}\right)}{\mathrm{PAT}_{\mathrm{e}}}\right]$ \\
Condensing & $Q_{c}=\dot{m}_{r}\left(h_{2}-h_{3}\right)$ & $=\dot{m}_{r}\left[\left(s_{3}-s_{2}\right)\right.$ \\
& & $\left.-\frac{\left(h_{3}-h_{2}\right)}{\mathrm{PAT}_{\mathrm{c}}}\right]$ \\
Expansion & $h_{3}=h_{4}$ & $I_{c a p}=\dot{m}_{r}\left(s_{4}-s_{3}\right)$ \\
\hline
\end{tabular}

Additionally, COP can be obtained from the following.

$C O P=\frac{Q_{\text {evap }}}{p}$

To calculate the rate of irreversibility for the evaporator and condenser, we need to calculate the process average temperature PAT for evaporator and condenser. In terms of measurable thermodynamic variables, the PAT is given by[16]
PAT $=\frac{\int_{i n}^{o u t} d h}{\sum_{i=1}^{n} \frac{\int d h}{T}}$

The PAT can be defined as the temperature of the refrigerant were the process to be considered as an equivalent isothermal process, for purposes of calculating irreversibility. In particular, the PAT is not a measured temperature; rather it is the proper reference temperature for evaluating dissipative losses, [16].

Finally, $I_{s}$ can be defined as irreversibility of the system and it encompasses the irreversibility of evaporator, condenser, compressor, and capillary tube. It can be calculated as.

$I_{s}=I_{e}+I_{c}+I_{\text {comp }}+I_{\text {cap }}$

Where $I_{e}, I_{c} I_{c o m p}$, and $I_{c a p}$ are rate of the irreversibility of the evaporator, condenser, compressor, and capillary tube, respectively.

The data gathered by Mahmood [14] will be reused. Furthermore, the irreversibility analysis was established using a C++ code developed by (Al-Nadawi, 2019)[2], which is based on the lumped parameter model. The code was modified to make it suitable for water chiller system. The properties of the refrigerants, namely R22, R407C, R404A, and R134A were obtained from the published data by ASHRAE Hand Book [17] with curve fit equations obtained by using MATLAB [18] for all refrigerants.

\section{Results and discussion}

In this investigation, many parameters are used to assess the irreversibilities. These are water temperature, ambient temperature, mass flow rate of the refrigerants, $I_{s}, \frac{I_{S}}{Q e}$, the refrigerant type, and COP.

The variation of the irreversibility of the system with time and for different ambient and water temperature for R22, R407C, R404A, and R134A is shown in Figure 4. The results reveals that there has been a slight increase in the total irreversibility of the system during the first forty minutes and then it gradually decrease in the range of forty to seventy minutes. However, R404A show a decreasing trend at alltime points. Also, it can be concluded from the figure that R404A show the highest irreversibility among other refrigerants, whereas R134A show the lowest one. Additionally, it is obvious that ambient temperature plays a crucial role in increasing the total irreversibility of the system. That are, the highest irreversibilities for R22, $\mathrm{R} 407 \mathrm{C}, \mathrm{R} 404 \mathrm{~A}$, and R134A are at $35^{\circ} \mathrm{C}, 37^{\circ} \mathrm{C}, 37^{\circ} \mathrm{C}$, and $40{ }^{\circ} \mathrm{C}$, respectively. The general trend may be associated with refrigerant mass flow rate as shown in Figure 5.
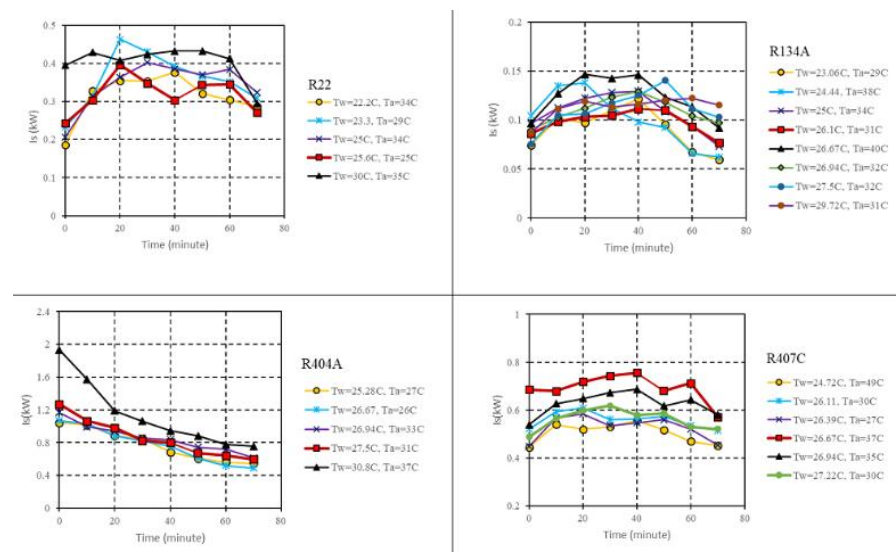

Figure 4. Variation of $I_{s}$ as a function of time for R22, R407C, R404A, and R134A.

Looking at Figure 5, it is apparent that the refrigerant mass flow rate decreases as the time of operation increases. 
This can be attributed to the fact that the water temperature decreases as the time of operation increases, and therefore, the refrigerant mass flow rate tends to decrease. Further, the highest refrigerant mass flow rate recorded with R404A, while the lower one recorded for R134A. This can be clearly explain the behaviour of total irreversibility as illustrated in Figure 4.
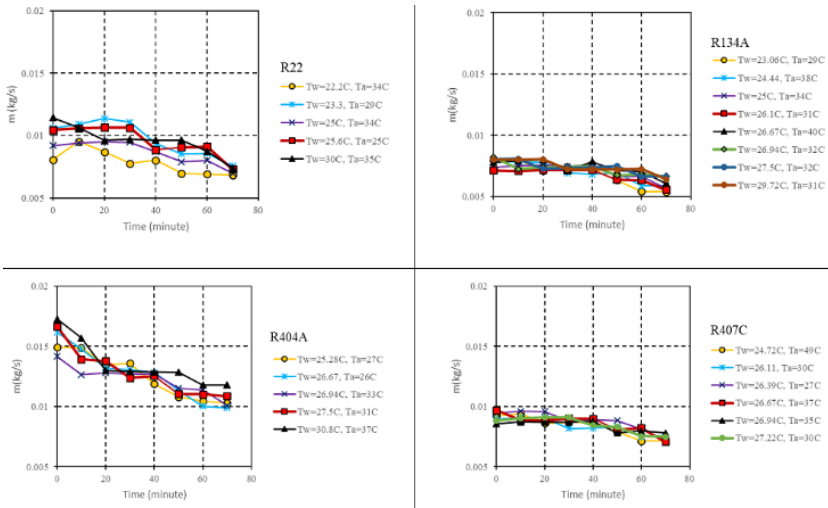

Figure 5. Variation of refrigerant mass flow rate as a function of time for R22, R407C, R404A, and R134A.

It would be interesting to consider the effects of combined $\frac{I_{S}}{Q e}$, Figure 6 . This term can be used to describe the relationship between $I_{S}$ and $Q_{e}$ with the increasing time. From the data in Figure 6, it is apparent that $\frac{I_{S}}{Q e}$ increased with increasing time for R22, R407C, and R134A. Contrarily, R404A show the opposite trend. What can be clearly seen in this figure is the dominance of $I_{S}$ which affects strongly the general trend, which also can be explained by Figure 4.
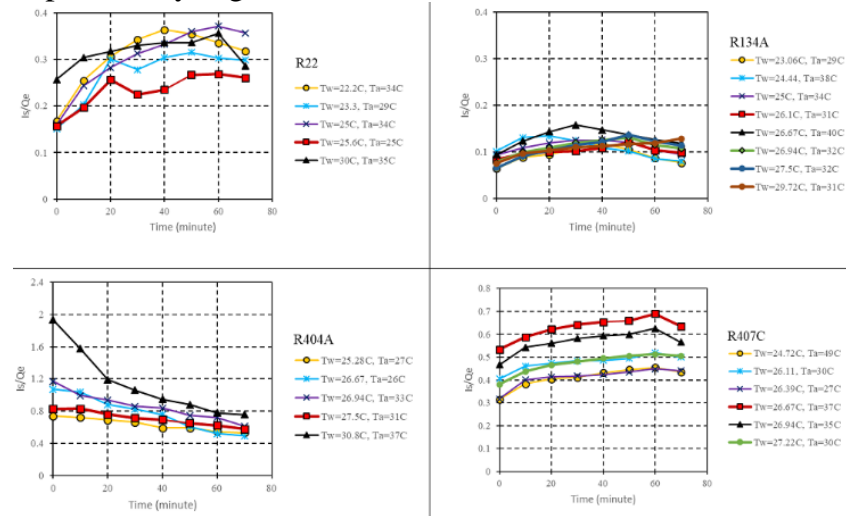

Figure 6. $\frac{I_{s}}{Q e}$ as a function of time for R22, R407C, R404A, and R134A.

Figure 7 presented the relationship between COP and time for R22, R407C, R404A, and R134A. This figure reveals that there has been a slight decline in COP for all refrigerants which may be associated with the effect of refrigerant mass flow rate. Nevertheless, it is clear that the COP of R22 is the highest among other refrigerants. This trend may have been caused by the fact that the system was originally designed to work with R22. Therefore, the system should be redesigned to meet the other refrigerant design criteria. Finally, we can conclude that R134A and R407C are a good replacement for R22 in terms of irreversibility analysis.

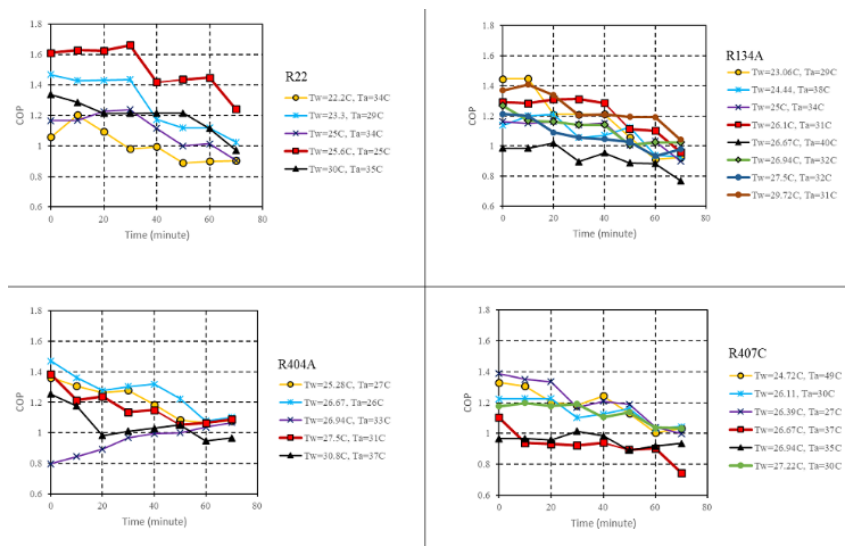

Figure 7. COP against time for R22, R407C, R404A, and R134A

\section{Conclusions}

The following conclusions can be drawn from the present investigation:

1. The total irreversibility of the system increases with time for R22, R 407C, R404A, and R134A.

2. R404A show the highest irreversibility, whereas R134A show the lowest one.

3. The total irreversibility increases at high water mass flow rate. Further, high ambient temperature increase the irreversibility of the system.

4. R134A and R407C are a good replacement for R22 in terms of irreversibility analysis.

\begin{tabular}{ll}
\hline Nomenclature & \\
\hline ODP & Ozone depletion potential. \\
COP & Coefficient of performance. \\
GWP & Global warming potential. \\
$\mathrm{h}$ & Specific enthalpy, $\mathrm{kJ} \mathrm{kg}^{-1}$ \\
$\mathrm{Q}$ & Discrete value, Capacity, $\mathrm{kW}$ \\
$\dot{m}$ & Mass flow rate, $\mathrm{kg} \mathrm{s}^{-1}$ \\
$\mathrm{p}$ & Power, $\mathrm{kW}$ \\
$\mathrm{V}$ & Voltage \\
$\mathrm{I}$ & Ampere \\
$\mathrm{I}$ & Irreversibility \\
$\mathrm{s}$ & Specific entropy, $\mathrm{kJ} \mathrm{kg}^{-1} \mathrm{~K}^{-1}$ \\
$\mathrm{PAT}$ & Process average temperature \\
$\mathrm{P}$ & Pressure, MPa \\
$\mathrm{T}$ & Temperature, $\mathrm{K}$ \\
$\mathrm{Cp}$ & Specific heat at constant pressure, $\mathrm{kJ} \mathrm{kg}^{-1} \mathrm{~K}^{-1}$ \\
\hline Greek Symbols & \\
\hline$\varnothing$ & Power phase angle \\
\hline Subscripts & \\
$\mathrm{e}$ & Evaporator \\
4 & Evaporator inlet \\
1 & Evaporator outlet \\
$\mathrm{w}$ & Water \\
$\mathrm{w}_{\mathrm{i}}$ & Water in \\
$\mathrm{w}_{\mathrm{o}}$ & Water out \\
$\mathrm{r}$ & Refrigerant \\
$\mathrm{s}$ & System \\
comp & Compressor \\
cap & Capillary \\
$\mathrm{c}$ & Condenser \\
2 & Condenser inlet \\
3 & Condenser outlet \\
\hline & \\
\hline & \\
\hline
\end{tabular}




\section{References:}

[1] Handbook for the Montreal Protocol on Substances that Deplete the Ozone Layer, 14th ed. Nairobi, Kenya: United Nations Environment Programme, 2020.

[2] A. K. Al-Nadawi, "Irreversibility Analysis of R407A and R407C As Alternatives of R22 in Window Type Air Conditioner," Arch. Thermodyn., vol. 40, no. 4, pp. 129-150, 2019.

[3] K. Zhang, Y. Zhu, J. Liu, X. Niu, and X. Yuan, "Exergy and Energy Analysis of a Double Evaporating Temperature Chiller," Energy Build., vol. 165, pp. 464-471, Apr. 2018.

[4] A. Mota-Babiloni, J. M. Belman-Flores, P. Makhnatch, J. Navarro-Esbrí, and J. M. BarrosoMaldonado, "Experimental Exergy Analysis of R513A to Replace R134a in a Small Capacity Refrigeration System," Energy, vol. 162, pp. 99110, Nov. 2018.

[5] H.-J. Tang, S.-X. Zheng, Y.-T. Yang, C. Wang, Y.X. Zhao, and S.-Y. He, "Energy and Exergy Analysis of a Refrigeration System with Vapor Injection Using Reciprocating Piston Compressor," in 2nd International Conference on Sustainable Development (ICSD 2016), 2016, pp. 436-439.

[6] R. Ben Jemaa, R. Mansouri, I. Boukholda, and A. Bellagi, "Energy and Exergy Investigation of R1234ze as R134a Replacement in Vapor Compression Chillers," Int. J. Hydrogen Energy, pp. 1-11, 2016.

[7] A. Dudar, D. Butrymowicz, K. Smierciew, and J. Karwacki, "Exergy analysis of operation of twophase ejector in compression refrigeration systems," Arch. Thermodyn., vol. 34, no. 4, pp. 107-122, 2013.

[8] S. Fukuda, H. Kojima, C. Kondou, N. Takata, S. Koyama, and H. Kojima, "Experimental Assessment on Performance of a Heat Pump Cycle Using R32/R1234yf and R744/R32/R1234yf," in International Refrigeration and Air Conditioning Conference, 2012, pp. 1-8.
[9] J. U. Ahamed, R. Saidur, H. H. Masjuki, and S. Mehjabin, "Prospect of hydrocarbon uses based on exergy analysis in the vapor compression refrigeration system," in 2011 IEEE 1st Conference on Clean Energy and Technology, CET 2011, 2011, pp. 67-70.

[10] C. Stanciu, A. Gheorghian, D. Stanciu, and A. Dobrovicescu, "Exergy analysis and refrigerant effect on the operation and performance limits of a one stage vapor compression refrigeration system," termotehnica, pp. 36-42, 2011.

[11] J. A. Shilliday, S. A. Tassou, and N. Shilliday, "Comparative Energy and Exergy Analysis of R744, R404A and R290 Refrigeration Cycles," Int. J. LowCarbon Technol., vol. 4, no. 2, pp. 104-111, Jun. 2009.

[12] H. Liang and T. H. Kuehn, "Irreversibility Analysis of a Water-To-Water Mechanical-Compression Heat Pump," Energy, vol. 16, no. 6, pp. 883-896, 1991.

[13] A. K. Al-Nadawi, "Experimental and Theoretical Study of R407C and R407A as an Alternative of R22 Refrigerant in a Window Type Air Conditioner Baghdad-Iraq," Al-Mustansiriya University, 2010.

[14] D. M. N. Mahmood, "Experimental and Theoretical Evaluation for the Evolution in Alternatives Applications in Water Chillers," Al-Mustansiriya University, 2010.

[15] I. Lira, Evaluating the Measurement Uncertainty Fundamentals and Practical Guidance. London: Institute of Physics Publishing(IOP), 2002.

[16] J. M. Gordon and K. C. NG, Cool ThermodynamicsThe Engineering and Physics of Predictive, Diagnostic, and Optimization Methods for Cooling Systems. Cambridge International Science Publishing, 2001.

[17] Mark S. Owen, Ed., "Thermophysical Properties of Refrigerants," in 2009 ASHRAE Handbook Fundamentals (SI Edition), 2009.

[18] “MatLAB.” 2017. 